\title{
Presentation, Validation and Application of the DistrictHeating Modelica Library
}

\author{
Loïc Giraud, Roland Bavière, Mathieu Vallée, Cédric Paulus \\ Univ. Grenoble Alpes, INES, F-73375 Le Bourget du Lac, France \\ CEA, LITEN, 17, Rue des Martyrs, F-38054 Grenoble, France, roland. baviere@cea. fr
}

\begin{abstract}
District heating systems are a relevant solution for reducing $\mathrm{CO} 2$ emissions, especially in dense areas with older buildings. However, due to the heavy investment costs, there is a great interest in simulation and software solutions to reduce distribution losses, limit the overuse of peak generators and optimize the use of storage capacities. In this paper, we describe how we designed, validated and used a library of fast, precise and robust components for district heating systems. Among other results, we could reduce the number of equations in some components by a factor of 40 and demonstrate more than $10 \%$ reduction in heat losses on a sample application.
\end{abstract}

Keywords: district heating, physical modeling, dynamic simulation, supply temperature optimization

\section{Introduction}

In French urban area, residential buildings currently account for $60 \%$ of the total energy consumption. This sector is also responsible for a large amount of carbon dioxide emissions. Most of this consumption is due to space heating and domestic hot water production. Following the recommendations of the "Grenelle Environment Round Table", France is tied up to divide by four all emissions of greenhouse gases by 2050 . New energy solutions must therefore be searched for the building sector. Generalizing standards of low consumption in new housings (e.g. RT2012 building code) as well as setting up incentives for building retrofitting can only be considered as long term measures since the renewal rate of existing buildings is limited to $1 \%$. On the other hand, district heating systems may already play a role since they are generally well established in dense urban area. Such system may massively increase the share of renewable and recovery energies, especially in urban areas where the use of decentralized systems is problematic or impossible. These context elements explain why France is currently experiencing a new age of development for district heating networks.

In France, large well-established district heating systems are continuing their extension while many small networks are being built. However, due to the heavy investment costs of such systems, there is a great interest in simulation and energy planning software solutions leading to the reduction of distribution losses, limiting the overuse of peak generators and optimizing the use of centralized and decentralized storage capacities. In the Modelica community, previous work have been investigating issues related to short-term production planning in district heating networks (Velut et al, 2014). Our research group is currently involved in several research programs devoted to the definition of optimal operation of district heating systems. In this context we have been working on the development of computationally efficient and accurate dynamic simulation capabilities in order to propose and evaluate advanced control strategies.

The beginning of the present research program in January 2014 was devoted to the selection of an appropriate simulation platform, for instance able to host flexible model development. We have carried comparative studies of various candidates and the details of this analysis can be found in (Giraud et al, 2014). This work has led us to the conclusion that the equation-based object-oriented language Modelica along with the simulation platform Dymola was the most adapted tool for our application. This has led us to develop a Modelica component model library that we named DistrictHeating.

The purpose of this paper is to present the DistrictHeating library and to show how accurate modelling of a district heating network can be used as a basis to develop efficient control strategies. Section 2 first describes the structure and some of the models contained in the DistrictHeating library. Since the experimental validation of such models is an important issue, the validation process applied for the pipe model is entirely described in section 3. In section 4, we describe the optimization of a temperature control strategy in a virtual district heating network, designed to reproduce the behavior of a small part of the district heating network in Grenoble, France. Consumers are simulated using actual heat load profiles observed in the Grenoble main district heating system. Two supply temperature control strategies, a standard and an 
optimized strategy, are compared in order to show the potential of energy savings.

\section{Library for district heating system modelling and simulation}

The current version of the DistrictHeating library contains several models intended to provide solutions for dynamic simulation of district heating and cooling systems. The modelling scope of the library is limited to systems using liquid water as the heat carrier fluid. As a consequence, the applicability of the developed models is restricted to cases where the fluid can be considered slightly- or in-compressible and poorly- or non-expandable.

\subsection{Compatibility}

Our DistrictHeating library relies on many modelling solutions provided by the version 3.2.1 of the Modelica Standard Library. For instance the FluidPort connectors defined in the Modelica.Fluid library (Franke et al, 2009) and relying on stream variables are used. The DistrictHeating library can thus handle the flow-reversals which can occur for instance in meshed networks or networks with multiple supply points. The HeatPort connectors of the Modelica.Thermal library are also used. The modelling of one-dimensional thermo-fluid flows in piping network within the DistrictHeating library is compliant with the Modelica.Media package. We have also chosen to base our work on the Modelica.Fluid library since it allows modeling fluids with multiple trace substances. This feature can for instance be used to dynamically track the influence area of various production plants on the same heat grid. The compatibility of our model developments with other already existing, well documented and open-source modelica libraries such as Buildings (Wetter et al, 2014) was also an important design criterion.

Although the DistrictHeating library is limited to our internal use for now, we can envision a wider diffusion of some components under an open-source license in the future.

\subsection{Structure of the DistrictHeating library}

The DistrictHeating library is composed of several packages containing model solutions for various components of a district heating system such as preinsulated pipe, pump, substation, heat generator... The most important packages of our library are described in the following sections.

\subsection{Package Fluid}

The Fluid package provides components to model onedimensional fluid flow in networks (see Figure 1).

The models representing one-dimensional pipes are gathered in the Pipes sub-package. The piping network of a district heating system is generally composed of two identical parts namely the supply and the return networks. The twin-pipe configuration ${ }^{1}$ apart, each piping element is composed of a pipe and a surrounding cylindrical thermal insulation. In some cases, it is necessary to account for the internal heat exchange between the supply and the return networks. In all cases, heat losses towards the surrounding environment should be accounted for. To ease the data collection burden for end-users we have developed a model representing a pair of pre-insulated pipes (see Figure 2) relying on geometrical parameters and solid properties available from vendors. We have collected such models in the PipeShopCatalogue sub-package.

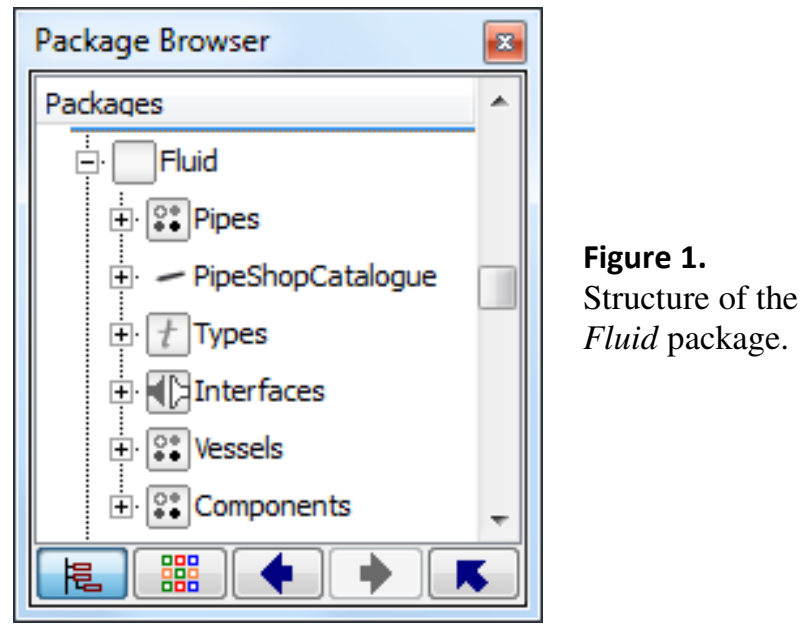

A model of tank with variable level is not useful for our application. We also found that head losses experienced by the fluid at inlet/outlet connections of a tank were generally negligible for our application. Following these two considerations, we have decided to introduce a Vessels sub-package in our library. This sub-package can be considered as a simplified version of the Modelica.Fluid.Vessels package.

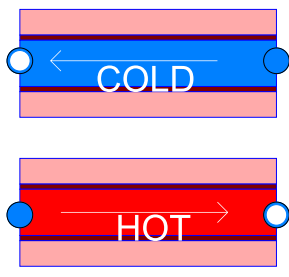

Figure 2. Icon representing a pair of pre-insulated pipes.

Some systems are built from $\boldsymbol{n}(\boldsymbol{n} \in \mathbb{N}, \boldsymbol{n}>1)$ identical components (eg tube, pump ...) in parallel. For clarity reasons, let us only consider here the case of $\boldsymbol{n}$ tubes in parallel. When the operating conditions are similar for each tube, it is tempting to model the system relying on one elementary pipe affected with a weight of $\boldsymbol{n}$. The weight feature is for instance available in the DynamicPipe model of the Modelica.Fluid library through the use of the nParallel parameter. However, implementing this

\footnotetext{
${ }^{1}$ Configuration with two pipes in the same casing.
} 
feature has led to the modification of 11 files within the Modelica.Fluid.Pipes sub-package. To ease the maintenance of our code, we have searched for a less intrusive mean of programming this feature. A FlowDivider and a HeatDivider model were developed for this purpose. These models are intended to be positioned at the interfaces of the system built from $\boldsymbol{n}$ identical components. Both models consist of a pair of ports introducing a division (respectively a multiplication) of the flow variables (mass flow-rate and heat flow-rate) in the positive (respectively negative) flow direction. Of course, the pressure drop and the accumulation terms for mass and energy are set to zero. These models are part of the Components subpackage.

\subsubsection{Focus on the pipe model}

Heat is convected at a velocity typically ranging from $0.05 \mathrm{~m} / \mathrm{s}$ to approximately $2 \mathrm{~m} / \mathrm{s}$ and over distances of a few to several tens of kilometers in a district heating network. As a consequence, a temperature change initiated at a production plant reaches far endconsumers with a delay that can exceed several hours. In order to limit heat losses throughout the distribution network, this delay must be correctly evaluated in order to overheat the network only when the thermal demand is high. Defining an optimal operation strategy for a district heating system therefore requires the use of a reliable pipe model correctly accounting for the temperature propagation dynamics. In order to define the best possible combination between accuracy and numerical performance we have developed two different numerical models to represent a district heating distribution pipe. Both models share common equations to express the momentum and mass balances across the pipe.

Several friction models ranging from linear relations to detailed laws accounting for the laminar-turbulent transition and the effect of roughness are available for end-users. However, a quadratic law fitted to data corresponding to nominal conditions and linearized in the laminar regime generally represents the best comprise between accuracy and numerical performance for district heating applications.

The following simplified equation derived from the first law of thermodynamics for open systems is used to express the 1-D fluid's energy conservation:

$$
A \cdot \rho \cdot c_{p}\left(\frac{\partial T}{\partial t}+v \frac{\partial T}{\partial x}\right)=\mathrm{A} \cdot \lambda \frac{\partial^{2} T}{\partial x^{2}}+\dot{Q}
$$

where $\boldsymbol{A}$ stands for the cross-sectional pipe area, $\boldsymbol{\rho}, \boldsymbol{c}_{\boldsymbol{p}}$ and $\lambda$ respectively stand for the fluid density, specific heat capacity and thermal conductivity, $\boldsymbol{T}, \boldsymbol{v}$ and $\dot{\boldsymbol{Q}}$ stand for the fluid temperature, velocity and the wall to fluid heat transfer-rate. As shown, in the validation section, the first term of the right hand side of Equation (1) can generally be ignored.
Equation (1) is a partial derivative equation (PDE) that requires the use of a numerical method to convert it in a form solvable by a computer program. We developed two pipe models based on two different numerical methods. These methods are respectively referred as the "element-" and the "node-" method in the Danish scientific literature devoted to the modelling of temperature propagation within a district heating network (Benonysson, 1991; Gabrielaitiene et $a l, 2008)$.

\section{The ElementPipe model}

In the first model, Equation (1) is spatially discretized using a finite volume method (Patankar, 1980). Relying on a collection of axially distributed non overlapping control volumes, Equation (1) is transformed into a system of ordinary differential equations that can be solved by a MODELICA tool. Each equation results from an integration of Equation (1) over an elementary control volume where the fluid temperature is assumed to be uniform.

The finite volume method is implemented using the DynamicPipe from the Modelica.Fluid library as a basis. The following modifications applied to the original DynamicPipe model are worth noting. Firstly, only one balance equation per pipe is considered for mass and momentum regardless of the number of control volumes used for the energy equation. Secondly, the discretization scheme for the convection term in the energy balance equation has been upgraded from an Upwind-Difference Scheme to the higherorder Quadratic Upstream Interpolation for Convective Kinematics (QUICK) scheme (Leonard, 1979). The QUICK scheme intends to limit artificial (also called numerical) diffusion. Thus, for a given accuracy level, larger mesh sizes can be used thereby improving the numerical efficiency of the model.

\section{The NodeMethodPipe model}

In the second model, Equation (1) is integrated along a fluid's particle path line following the method of characteristics (Wylie et al, 1978). Along the path line, namely a characteristic curve, the PDE becomes an ordinary differential equation which can be natively integrated within a Modelica based computer program.

Following this method, it can be shown that the outlet temperature of a pipe can be inferred from a past inlet value according to the following equations (see (Giraud, 2015) for details):

$$
T_{\text {out }}^{*}(t)=\left(T_{\text {in }}(t-\tau)-T_{\text {ext }}\right) e^{-\frac{\tau}{\tau_{t}}}+T_{\text {ext }}
$$

where $\boldsymbol{T}_{\boldsymbol{i n}}(\boldsymbol{t}-\boldsymbol{\tau})$ represents the pipe inlet temperature at the past instant $\boldsymbol{t}-\boldsymbol{\tau}, \boldsymbol{\tau}$ is the transportation time, $\boldsymbol{T}_{\text {ext }}$ is the external temperature, $\boldsymbol{\tau}_{\boldsymbol{t}}$ is a heat loss characteristic time and $\boldsymbol{T}_{\text {out }}^{*}(\boldsymbol{t})$ is the pipe outlet temperature obtained by neglecting the heat capacity of the tube.

$\boldsymbol{\tau}$ is determined using (3) where $\boldsymbol{L}$ is the length of the pipe and $\boldsymbol{v}$ is the mean fluid velocity: 


$$
\int_{t-\tau}^{t} v(s) d s=L
$$

$\boldsymbol{T}_{\text {out }}^{*}(\boldsymbol{t})$ is then modified to account for the heat stored in the steel tube by assuming that the heat capacity of the whole tube is gathered at the outlet of the pipe and that the fluid and tube temperatures are equal:

$$
m_{a} \frac{d T_{\text {out }}}{d t}=\dot{m} c_{p_{f}}\left(T_{\text {out }}-T_{\text {out }}^{*}\right)
$$

where $\boldsymbol{T}_{\text {out }}$ is the fluid outlet temperature, $\boldsymbol{m}_{\boldsymbol{a}}$ is the mass of the steel tube and $\dot{\boldsymbol{m}}$ and $\boldsymbol{c}_{\boldsymbol{p}_{\boldsymbol{f}}}$ respectively stand for the fluid mass flow-rate and the fluid specific heat capacity.

Equations (2), (3) and (4) have been implemented using a combination of the delay(...) and the spatialDistribution(...) operators (Modelica Association, 2014).

Table 1. Relative number of equations of the DAE system for a model composed of a pre-insulated district heating pipe and two boundary conditions

\begin{tabular}{|l|l|l|}
\hline Model & Library & Rel. Nb. Eq. \\
\hline DynamicPipe & Modelica.Fluid & $100 \%$ \\
\hline ElementPipe & DistrictHeating & $32.7 \%$ \\
\hline NodeMethodPipe & DistrictHeating & $2.56 \%$ \\
\hline
\end{tabular}

Table 1 compares the size of the system of Differential Algebraic Equations (DAE) for different pipe models. The number of meshes for the finite volume models (namely DynamicPipe and ElementPipe) is chosen such that the obtained numerical results are close to the results yielded by the model based on the method of characteristics (namely NodeMethodPipe) for a typical district heating transient. As can be seen from Table 1 the number of DAEs for the ElementPipe model is smaller by a factor of 3 to that of the Modelica.Fluid DynamicPipe. More importantly a reduction by a factor of 40 is observed for the NodeMethodPipe. Accordingly, significant computational costs savings are observed with this model when compared to the DynamicPipe or even to the ElementPipe models. In district heating system simulations, these numerical optimizations do not have any accuracy impact, as shown in the validation section (Figure 5). The NodeMethodPipe is thus used for the application shown in section 4.

\subsection{Package Substation}

The heat transported throughout a district heating network is delivered to consumers by the mean of a substation. The purpose of the Substation package is to provide generic model solutions to represent the thermalhydraulic behavior of a district heating substation.
A substation is generally composed of a heat exchanger a control valve positioned on the primary side and a PI controller used to control the secondary outlet temperature (see Figure 3). Two distinct regimes must be considered to cover the operational conditions encountered in a district heating system. Firstly, in the thermal regime, the heat demand is satisfied and the primary mass flow-rate is entirely governed by the consumers' needs. On the other hand, in the hydraulic regime, the requested heat cannot be provided by the network. As a consequence, the control valve is fully opened and the primary mass flow-rate depends on the local pressure difference between the primary supply and return lines.

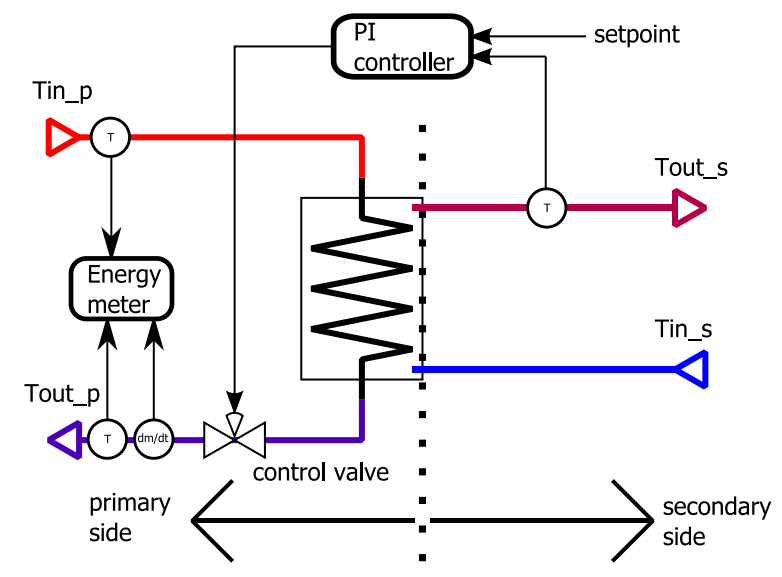

Figure 3. Schematic representation of a substation. $\boldsymbol{T i n}_{\boldsymbol{p}}$

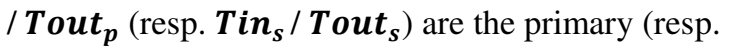
secondary) inlet / outlet temperatures.

We have developed several substation models in our library consisting of a control valve, a heat exchanger and a simplified controller. Details on this development work and on the validation procedure can be found in (Giraud et al, 2015). In the present paper, only the model representing the best compromise between accuracy and computational costs for our application will be described.

The purpose of a substation model is to cover simulation periods ranging from days to months. Consequently, the detailed dynamics of the PI controller can be ignored and the model may consider that the secondary outlet temperature always matches the set point value in the thermal regime. Correctly accounting for the heat exchanger behavior is a crucial part of the model. This part is based on the classical LMTD (Log Mean Temperature Difference) formulation (Shah et al, 2003):

$$
\text { Power }=U A \cdot L M T D
$$

where $\boldsymbol{U} \boldsymbol{A}$ stands for the global heat exchanger thermal conductivity.

The $\boldsymbol{L} \boldsymbol{M T} \boldsymbol{D}$ term reads: 


$$
L M T D=\frac{\Delta T_{1}-\Delta T_{2}}{\ln \left(\Delta T_{1} / \Delta T_{2}\right)}
$$

where $\Delta \boldsymbol{T}_{1}=\boldsymbol{T i n}_{p}-$ Tout $_{s}$ and $\Delta \boldsymbol{T}_{2}=$ Tout $_{p}-$ $\boldsymbol{T i n}_{\boldsymbol{s}}$ are the temperature differences at the two ends of the heat exchanger.

By considering that the solid/fluid heat transfers are similar for both sides of the heat exchanger and that the conduction in the solid part is negligible, $\boldsymbol{U A}$ can conveniently be expressed as:

$$
U A=\frac{U A_{\text {nom }} \cdot\left[\left(\dot{m} p_{\text {nom }}\right)^{-q}+\left(\dot{m} s_{n o m}\right)^{-q}\right]}{(m p)^{-q}+(\dot{m s})^{-q}}
$$

where $\boldsymbol{m} \boldsymbol{p}$ and $\boldsymbol{m} \boldsymbol{s}$ respectively stand for the primary and the secondary mass flow-rates. The nom subscript indicates nominal conditions values and $\boldsymbol{q}$ is a user-defined parameter generally of the order of 0.7 .
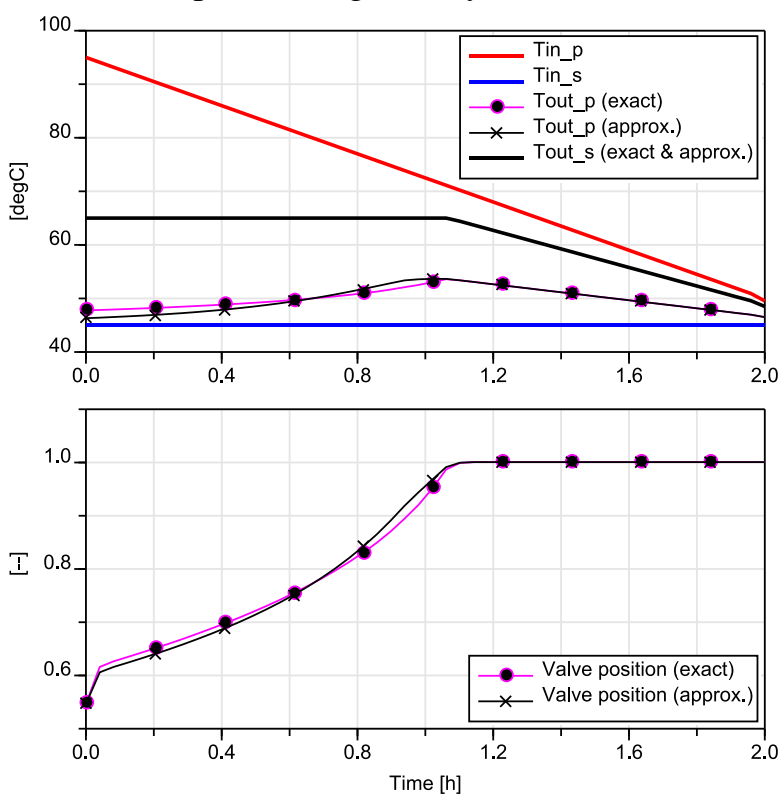

Figure 4. Evolutions of the temperatures (top) and valve position (bottom) for the exact and approximate substation models - for a decreasing network temperature.

Equations (5), (6) and (7) have been programmed in a Modelica model. When translated and solved by the DYMOLA FD01 2015 software, these equations lead to convergence and numerical stability issues, especially in the region where $\left|\Delta T_{1}-\Delta T_{2}\right| / \Delta T_{1} \ll 1$. As a workaround, the regularization method proposed in (Mattsson, 1997) was tested but the resulting model still suffered from numerical difficulties in the conditions of our application. Finally an alternative formulation, also linearized in the aforementioned region was implemented. In parallel, to improve numerical efficiency, we have searched for an approximate method relying on an explicit formulation that could be programmed in an algorithm section.
For the thermal regime, the heat exchanger behavior has been inferred from an explicit correlation between $\boldsymbol{m} \boldsymbol{p}$ and the inputs of the model, see (Giraud et al, 2015) for details. In the hydraulic regime $\boldsymbol{m} \boldsymbol{p}$ can be determined prior to the heat exchanger calculation. Equations (5), (6) and (7) can thus be solved explicitly is this regime.

Figure 4 compares the evolutions obtained with the "exact" and explicit (i.e. approximate) developed substation models. In the simulation scenario, the network temperature is progressively decreased while all other quantities are kept constant (power demand, network pressure difference, ...). Up to time $1.1 \mathrm{~h}$, the heat demand is satisfied and the substation model runs in the thermal regime. In this regime, both models predict an increase in $\boldsymbol{T o u t}_{\boldsymbol{p}}$ when $\boldsymbol{T i n}_{\boldsymbol{p}}$ decreases. This is a coherent behavior for a heat exchanger with a $\boldsymbol{U A}$ coefficient that is only slightly sensitive to mass flow-rates variations. However, since this Tout $_{\boldsymbol{p}}$ increase is limited, decreasing the supply temperature in a district heating network will generally lead to an overall heat losses improvement. In the hydraulic regime, Tout $_{\boldsymbol{s}}$ decreases and the consumers' heat demand cannot be fulfilled anymore. In summary, Figure 4 demonstrates a good qualitative behavior for our explicit substation model. We have also performed experimental validation relying on the analysis of temperature data recorded from instrumented district heating substations. Details of this validation results and procedures can be found in (Giraud et al, 2015).

\subsection{Other Packages}

The DistrictHeating Modelica library is also composed of many other packages providing solutions to model pumps, heat generator, stratified heat storage, conduction in multi-layers planar or cylindrical walls etc ... The thermophysical properties of the solid materials traditionally encountered in district heating and cooling systems are gathered in the SolidMaterials package.

\section{Validation}

In this section, we detail some of the experimental validation work that we have conducted for the pipe models described in section 2.3.1.

The accuracy of the different pipe models is assessed using the experimental data reported in (Ciuprinskas et al, 1999). The same experimental data have already been used in a similar validation work performed by (Gabrielaitiene et al, 2008). The measurements reported in (Ciuprinskas et al, 1999) have been obtained by triggering a temperature wave at a production plant of the Vilnius district heating network. The experiment has been conducted at the end of the heating season, when heat demand is low and network mass flow-rate is almost constant. Temperature measurements have been positioned at 
both ends of an horizontal $470 \mathrm{~m}$ in length pre-insulated pipe. Details on this case study and on the parameters that have been considered to build the corresponding model can be found in (Ciuprinskas et al, 1999; Gabrielaitiene et al, 2008).

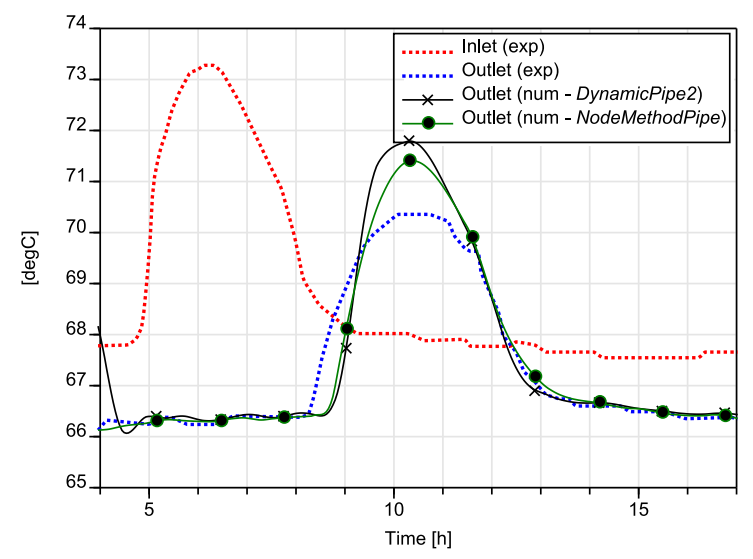

Figure 5. Numerical vs. experimental comparison of the temperature evolutions at both ends of an horizontal district heating pre-insulated pipe, $470 \mathrm{~m}$ in length.

Figure 5 plots the experimental and the numerical results for two pipe models of our library, namely the ElementPipe and the NodeMethodPipe (see section 2.3.1 for details). This figure firstly shows that heat losses are correctly evaluated by the models since the temperature evolutions at the outlet are all equivalent. However, the numerical predictions slightly differ from the experimental evolutions concerning the peak temperature and the time at which the outlet temperature starts to rise. These errors exceed the experimental uncertainties (not shown here) reported in (Ciuprinskas et al, 1999). The same findings were also reported for comparable pipe models developed in a non-modelica environment (Gabrielaitiene et al, 2008).

In (Gabrielaitiene et al, 2008) it is postulated that the aforementioned numerical errors could originate from the fact that turbulent axial dispersion is significant in the conditions of the study yet this phenomenon it is not accounted for by the models. Consequently, we have implemented a simplified version of the thermal diffusion and dispersion models proposed by (Drouin, 2010) in our ElementPipe model. This did not significantly improve our numerical predictions. We have also analyzed the potential impact of bends and other singularities on the temperature propagation dynamics by following the models proposed in (Park et al, 1971). Again, this did not improve our numerical predictions. We have also carried a sensitivity analysis on several uncertain experimental parameters (e.g. wall capacity, fluid/solid heat transfer coefficient, thermal dispersion coefficient ...). Within the considered variation ranges, it was not possible to significantly reduce the numerical errors.

Water tests on thermal stratification in a long horizontal pipe subject to transient inlet conditions were reported in (Tenchine et al, 2014). The purpose was to validate criteria for the prediction of occurrence and amplitude of thermal stratification in a simple horizontal pipe. The variation ranges regarding the experimental conditions explored in (Tenchine et al, 2014) are compatible with the experimental conditions of the present validation work in terms of pipe diameter, fluid mean velocity, amplitude of the inlet temperature transient but also Reynolds, Peclet, and Richardson dimensionless numbers. When applied to the district heating experimental conditions analyzed here, the criteria proposed in (Tenchine et al, 2014) suggest that the maximum cross-sectional temperature difference could reach $50 \%$ of the inlet temperature wave amplitude. In other words, thermal stratification has probably occurred in the experiments reported in (Ciuprinskas et al, 1999). This would explain why the time at which the outlet temperature starts to rise is significantly under-predicted by the pipe models of our library which all rely on the assumption of flat velocity and temperature profiles. Since, the operational conditions leading to potential occurrence of thermal stratification in a district heating network (very low mass flow-rates and steep temperature changes) are

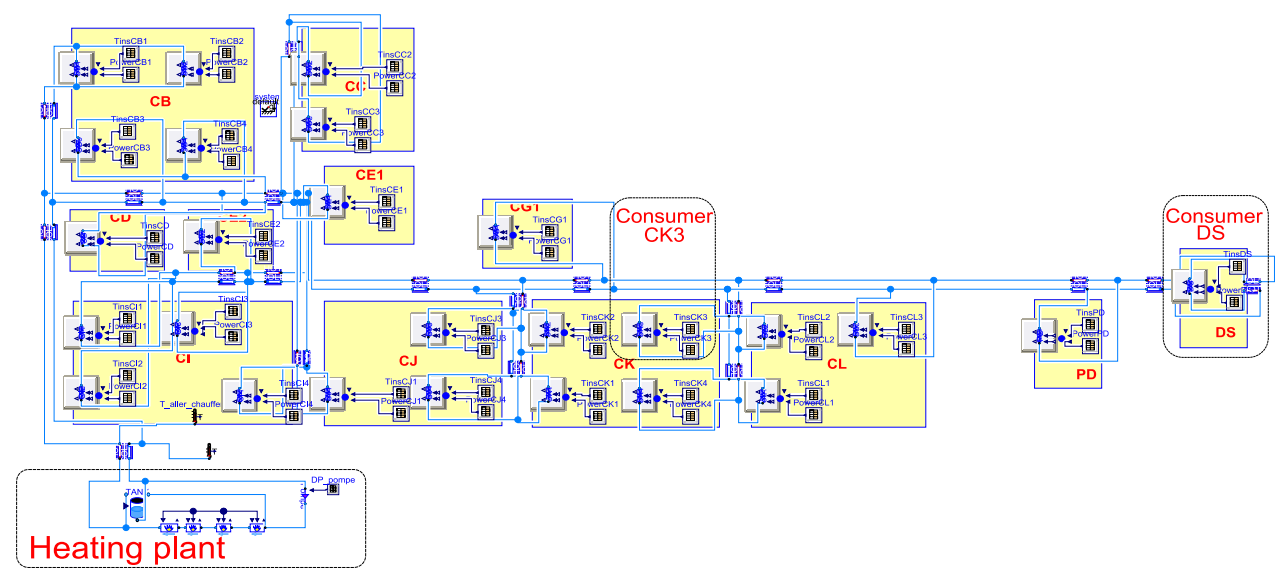

Figure 6. Layout of the sample district heating network, with 26 consumers and one heating plant. 
very rare, we have decided to not include such a phenomenon in our pipe models. However, to complement the validation procedure exposed in the present paper, a dedicated experiment will be conducted in the Grenoble district heating network. More realistic experimental conditions will be targeted in order to exclude the potential occurrence of thermal stratification.

In summary, the numerical predictions produced by the available pipe models of the DistrictHeating library for a typical district heating transient are comparable to those obtained by other research group relying on nonModelica tools (Gabrielaitiene et al, 2008). However, when compared to the experimental data reported in (Ciuprinskas et al, 1999), a slight numerical error is observed. By analysing the possible origins for this error, we have found that thermal stratification might have biased the temperature transport measurements performed in (Ciuprinskas et al, 1999). Further experimental work would be required to firmly conclude on this issue.

\section{Application: optimized supply temperature}

After presenting the library and its validation, we now present a concrete application of the library on a sample district heating network. In this section, we first give an overview of the realistic virtual district heating network we consider. We then describe the issue of choosing the supply temperature and the standard control law generally used in industry. We then show how the precise, fast and robust simulation obtained with the DistrictHeating library makes it possible to quickly obtain optimized supply temperatures results.

\subsection{Overview of the virtual district heating network}

Figure 6 depicts the virtual district heating network we use for this study. The mesh-free network layout considered in the virtual network originates from an extension project of the main district heating network in Grenoble, France. However, since the characteristics of the buildings in this new district are not yet available, we reconstructed heat load profiles based on historical data from other existing buildings in Grenoble.

In order to make this simulation as realistic as possible, we dimensioned the various virtual components carefully, taking into account the following constraints. Firstly, the buildings' profiles are chosen in order to respect the usual distribution of district heating clients in France, composed of $58 \%$ households, 36\% services and $6 \%$ industries. Secondly, the substation models are dimensioned according to the local rules stating that the dimensioning load must be deliverable to consumers at a pressure difference of 1 bar and a heat exchanger temperature difference of $110 \mathrm{~K}$ (i.e. $\operatorname{Tin}_{p}-\operatorname{Tin}_{s}=$ $110 \mathrm{~K})$. Finally, the pipes internal diameters are chosen within the range DN65 to DN350 in order to limit the maximal fluid velocity to $1.5 \mathrm{~m} / \mathrm{s}$. This leads to a maximum heat transportation time between the boiler and the far end consumer of 3 hours in the operating conditions investigated in the present work. The various piping elements of the virtual district network are modelled using the NodeMethodPipe Table 1.
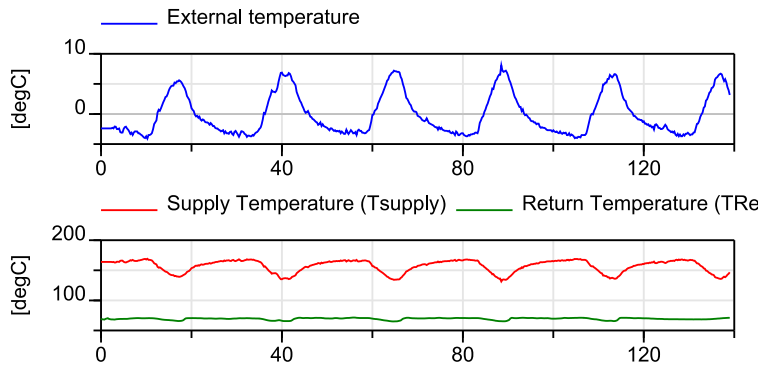

_ Maximum valve position (POS) in the network

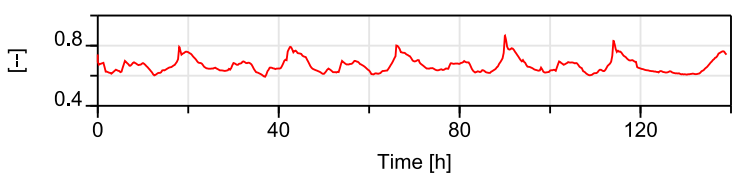

Figure 7. Evolution of the main variables over the simulation period (08/12/2013-13/12/2013).

The global heat losses coefficient of the network is adjusted to limit the relative losses to $10 \%$ of the distributed energy during typical winter days.

The simulation period covers five consecutive days of December 2013 characterized by a cold yet sunny anticyclonic weather with daily temperature variations ranging between $-4.1{ }^{\circ} \mathrm{C}$ and $+8.1{ }^{\circ} \mathrm{C}$. During this period, heat load patterns are regular, allowing for a particularly clear analysis.

\subsection{Standard control of the supply temperature}

In such a district heating network, correctly choosing the supply temperature at the heating plant is a good way of improving the efficiency of the whole system. On the one hand, the supply temperature must be high enough for substations to deliver the required power to customers. One the other hand, choosing higher supply temperatures increase heat losses during the transport, especially as transport times also tend to increase with higher temperatures. Perfectly optimizing the choice of the supply temperature would require taking into account the interdependencies between temperatures and transportation times for all consumers, as well as predicting the power requirements of all consumers over a few hours. In practice, even for a small network with a few dozen consumers, such a direct control proves impractical, both in terms of computation and in terms of investment costs for measurements. 
As a consequence, the standard supply temperature control strategy uses an indirect solution, based on a static heating curve. With this strategy, the supply temperature is chosen as a linear function of the external temperature. The linear dependency between the supply and external temperatures can be adapted so that the maximal opening of the control valves never exceeds a given threshold, leaving a security margin for extreme cases.

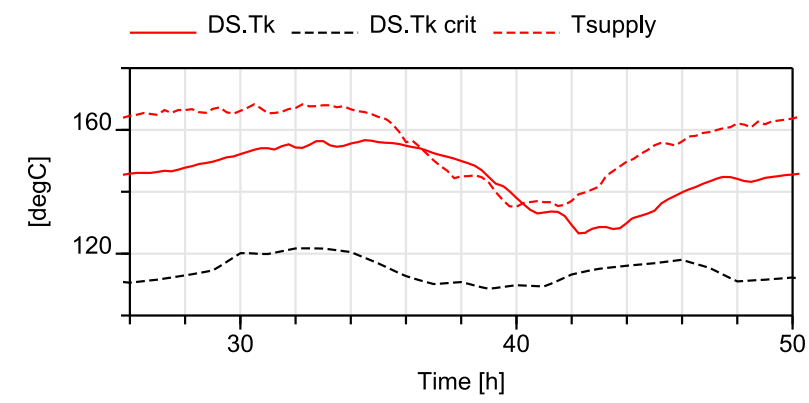

Figure 8. Supply temperature, network temperatures $\boldsymbol{T}_{\boldsymbol{k}}$ and critical temperatures $\boldsymbol{T}_{\boldsymbol{k}}^{\text {crit }}$ for consumer DS.

Using the virtual district heating network described above, we applied a standard supply control strategy, adapted so that the maximal opening of the control valves is limited to $85 \%$ during the simulation period.

Figure 7 depicts the evolution of the main variables over the simulation period: the external temperature; the supply and return temperatures as well as the mass flow rate at the heating plant; and the maximal opening of control valves.

Since the choice of the supply temperature is only indirectly related to the heat loads at the consumer, and especially does not take into account transport times, we can observe that this standard control strategy leads to large variations of the opening of the control valves. This behavior is consistent with the observations in a real DH system.

Looking at the consumer side, we can use the substation models for a finer analysis. Figure 8 depicts the network temperatures at a consumer, named DS (red), together with the supply temperature (red dashed). One indicator we can compute thanks to the detailed substation model is the critical temperature (black), i.e., the minimal temperature required to be able to satisfy consumer demand. On these figures, we can see the actual network temperature is much higher than the critical temperature. For instance it can be about $150^{\circ} \mathrm{C}$ when only $120^{\circ} \mathrm{C}$ would be sufficient.

\subsection{Optimizing the supply temperature}

For each substation, we can compute the critical temperature, hereafter noted $\boldsymbol{T}_{k}^{\text {crit }}$, which corresponds to the minimal network temperature allowing to serve the power required by consumer $\mathrm{n}^{\circ} \boldsymbol{k}$. By taking into account transport times and heat losses, we can further compute the critical temperature at the heating plant according to the following equation:

$$
\begin{gathered}
T_{\text {sup }}^{\text {crit }}(t)=\max _{k, t^{\prime}}\left(T_{k}^{\text {crit }}\left(t^{\prime}\right)+\operatorname{losses}\left(\tau\left(t^{\prime}\right)\right)\right), \\
\forall t^{\prime} \text { such that } t=t^{\prime}-\tau\left(t^{\prime}\right)
\end{gathered}
$$

Choosing a supply temperature close to $\boldsymbol{T}_{\text {sup }}^{\text {crit }}(\boldsymbol{t})$ ensures that demand at consumer side will be satisfied at all time, while minimizing heat losses. For a given set of heat load predictions, we can obtain all the data to compute $\boldsymbol{T}_{\text {sup }}^{\text {crit }}(\boldsymbol{t})$ directly from the simulation results. However, since modifying the supply temperature also modifies mass flow rates, transport time, and thus network temperature, the selection of an optimized supply temperature is actually an iterative process.

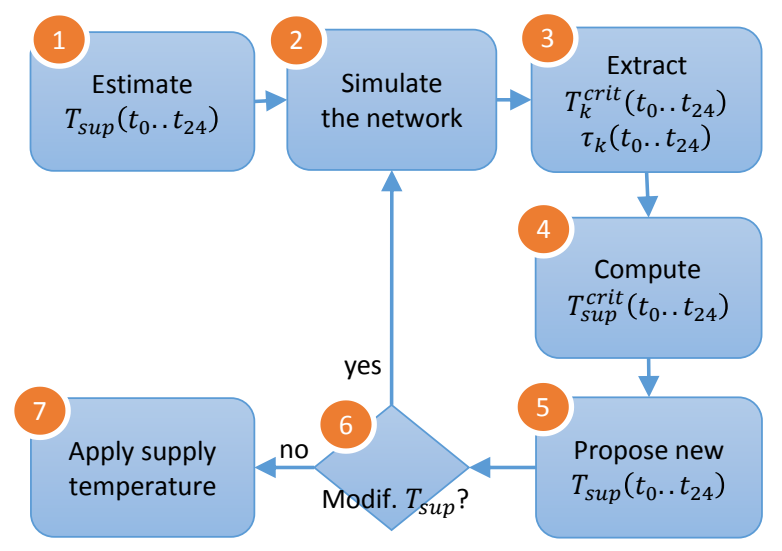

Figure 9. Computation of an optimized supply temperature, over a $24 \mathrm{~h}$ prediction horizon $\left(\boldsymbol{t}_{\mathbf{0}} . . \boldsymbol{t}_{\mathbf{2 4}}\right)$.

Figure 9 depicts the iterative process computing an optimized supply temperature:

1. Estimate an initial supply temperature schedule

2. Simulate the network using the Modelica DistrictHeating library

3. Extract critical temperatures and transport time for each consumers

4. Compute the aggregated critical temperature at the heating plant, taking into account individual critical temperatures and time delays (see Equation (8))

5. Choose a new supply temperature based on the aggregated critical temperature

6. Check if the supply temperature changed, and iterate to step 2 if necessary.

7. Once a solution is found, apply the supply temperature.

For our application, we implemented this algorithm in the Scilab scientific computing software (Scilab Enterprises, 2015), which communicates with Dymola for executing the Modelica simulation. This setting is close to the one adopted in previous work (Du et al, 2014), for optimizing dynamic hybrid energy systems. 

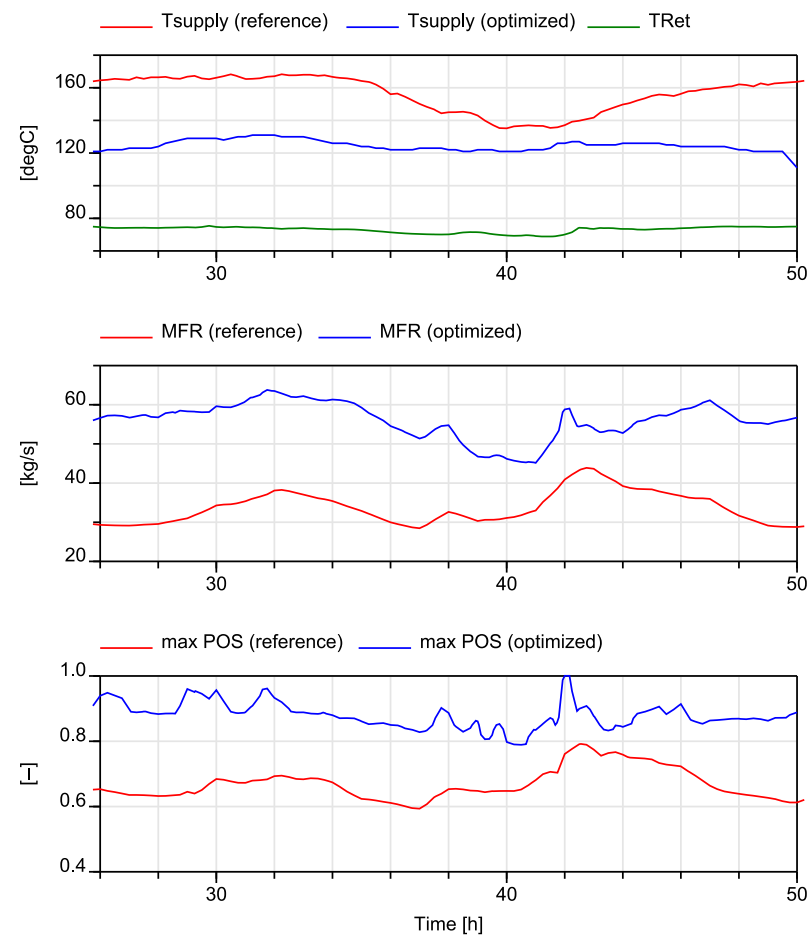

Figure 10. Main variables at the heating plant and maximal control valve opening, in the reference (red) and optimized (blue) cases. Return temperature at the heating plant (green) is similar in both cases.

\subsection{Comparison between standard and optimized supply temperature}

Figure 10 depicts the evolution of the main variables at the heating plant and the maximal control valve opening, both using the standard supply temperature (in red), and using the optimized supply temperature (in blue). In the upper figure we can see that the supply temperature can be reduced by up to $30^{\circ} \mathrm{C}$, without reducing the ability to meet the consumers demand. In Figure 11, we can see that the produced thermal power is slightly reduced, because of a reduction of heat losses. Over the period we consider, the total energy savings amount to about $18 \%$ of the total heat losses.

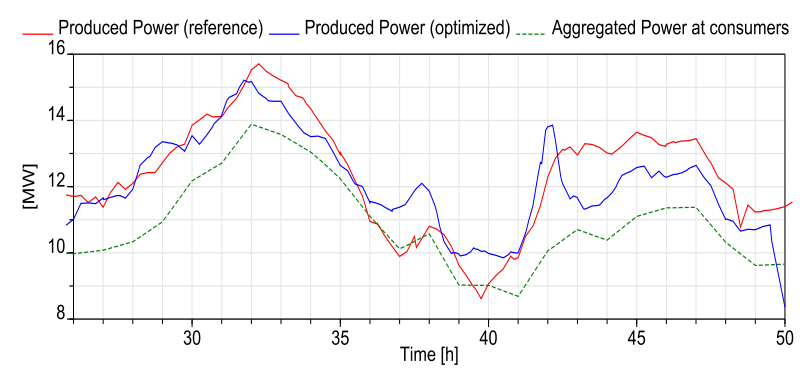

Figure 11. Produced thermal power in the reference (red) and optimized (blue) cases, and aggregated thermal power transferred to consumers (green).

Looking at the consumer side, Figure 12 clearly shows that the optimized network temperature (blue) is lower than using the standard supply temperature (red), and is closer to the critical temperatures (black dashed). We can also observe that the control valves operate between $70 \%$ and $95 \%$ opening, and with more dynamic variations, indicating a finer control.
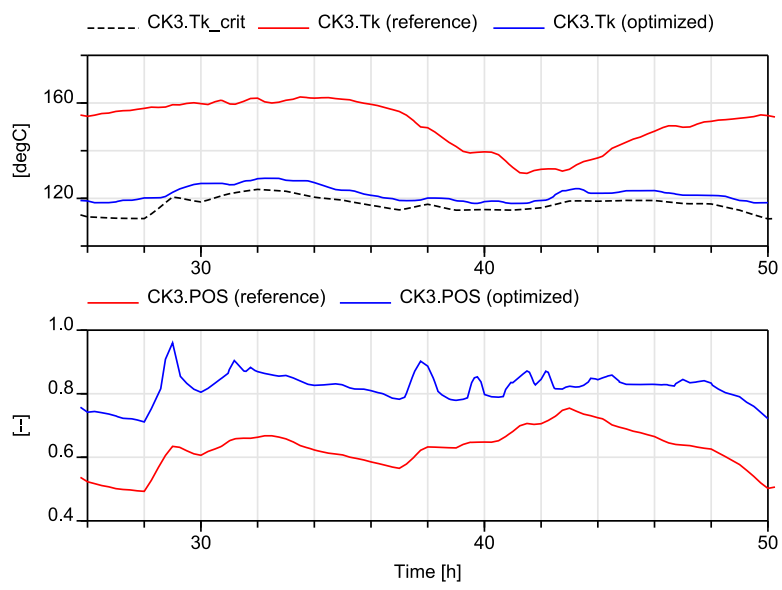

Figure 12. Results for consumer $C K 3$ in the reference (red) and optimized (blue) cases: network temperatures (top), control valve position (bottom).

\subsection{Discussion about heat load prediction}

We can notice that heat load prediction for consumers plays an important role in the optimization of the supply temperature. In the work presented above, incorrect predictions could lead to lower network temperatures at the substations, and prevent from meeting the heat demand at some points in time. To mitigate this constraint, we can note the following points:

1. Since critical temperatures at consumers $\boldsymbol{T}_{k}^{\text {crit }}$ are close to linearly related to the heat load at each consumer, we can propagate prediction uncertainties to the choice of a supply temperature. For instance, if there is $10 \%$ uncertainty on the heat load prediction, increasing the optimized supply temperature by $10 \%$ of the typical temperature difference (approx. $1^{\circ} \mathrm{C}$ in most cases) would provide a good confidence to the network operator.

2. Even in case the network temperature is too low for meeting the heat demand during short periods of time, previous studies have shown that the thermal inertia of buildings ensure the inhabitants' comfort for at least a few hours (Kensby et al, 2015). In typical buildings, it is only after a few dozen hours that a reduction of heat power by $10 \%$ will lead to a reduction of internal temperature by $2{ }^{\circ} \mathrm{C}$.

3. To minimize prediction uncertainty, we can adopt a model-predictive control approach, in which the optimized supply temperature is updated on a regular basis with new predictions. Thanks to the rather slow dynamics of a district heating network, computing a new optimized supply temperature 
every 15 minutes is enough to guarantee a finegrained control.

\section{Conclusion}

In this paper, we describe how we designed, validated and used DistrictHeating, a library of fast, precise and robust Modelica components for district heating systems. In this library, we designed the components by iteratively improving standard components in order to reach a good balance between the precision and execution time of the simulation. We then validated the components by comparing their behavior with experiments on real district heating networks.

Based on the DistrictHeating library, we could design an application for optimizing the supply temperature of a sample district heating network. Using an optimized supply temperature allows reducing heat losses by $18 \%$ compared to a standard strategy. This optimized strategy relies on accurate prediction of the heat load for consumers.

As a further step, we will develop a modelpredictive control approach for supply temperature optimization. In this approach, we can update heat load predictions regularly, and compute an optimized supply temperature for the next time slot accordingly. The improved simulation times of our library make it possible to adopt an update rate of a few minutes even for medium size systems (dozens of consumers), which is perfectly compatible with the slow dynamics of a district heating network. Using this approach, we plan to extend our study to a one-year simulation in order to estimate possible yearly savings on our test case.

\section{Acknowledgements}

The authors wish to thank Elise Le Goff, Nicolas Giraud and Philippe Clolot from CCIAG for their fruitful help in the realization of this study. We also would like to acknowledge the financial support of CCIAG for the joint research program and of ADEME for the PhD of Loïc GIRAUD.

\section{References}

A. Benonysson, "Dynamic Modelling and Operational Optimization of District Heating Systems," ISBN 8788038-24-6. PhD Thesis - Technical University of Denmark, Lyngby, 1991

K. Ciuprinskas and B. Narbutis, Experiments on heat losses from district heating pipelines. Energetika vol. 2 pp. 35 40, 1999

M. Drouin, "Modélisation des écoulements turbulents anisothermes en milieu macroporeux par une approche de double filtrage," PhD Thesis - Université de Toulouse, 2010 http://www.theses.fr/2010INPT0066

W. Du, H. Garcia and C. Paredis, "An Optimization Framework for Dynamic Hybrid Energy Systems", Proc. of the 10-th International Modelica Conference, Lund, Sweden, March 2014. DOI: 10.3384/ECP14096767
R. Franke, F. Casella, M. Otter, K. Proelss, M. Sielemann, and M. Wetter "Standardization of thermo-fluid modeling in Modelica.Fluid" in Francesco Casella, editor, Proc. of the 7-th International Modelica Conference, Como, Italy, September 2009. DOI: 10.3384/ecp09430077

I. Gabrielaitiene, B. Bøhm, and B. Sunden, "Evaluation of Approaches for Modeling Temperature Wave Propagation in District Heating Pipelines," Heat Transf. Eng., vol. 29, no. 1, pp. 45-56, 2008 DOI: 10.1080/01457630701677130

L. Giraud, R. Bavière and C. Paulus, "Modeling of Solar District Heating: A Comparison between TRNSYS and Modelica" Proc. of EuroSun 2014, Aix-les-Bains, France, 2014. DOI: 10.18086/eurosun.2014.19.06

L. Giraud, R. Bavière, C. Paulus, M. Vallée and J.-F. Robin "Dynamic Modelling, Experimental Validation and Simulation of a Virtual District Heating Network" in Proc. of the $28^{\text {th }}$ Int. Conf. on Efficiency, Cost, Optimization, Simulation and Environmental Impact of Energy Systems (ECOS), Pau, France, 30 ${ }^{\text {th }}$ June $-3^{\text {rd }}$ July 2015

J. Kensby, A. Trüschel, and J.-O. Dalenbäck, "Potential of Residential Buildings as Thermal Energy Storage in District Heating Systems - Results from a Pilot Test." Applied Energy, vol. 137 (January), pp. 773-781, 2015 DOI: 10.1016/j.apenergy.2014.07.026.

B. P. Leonard, "A stable and accurate convective modelling procedure based on quadratic upstream interpolation" Comput. Methods Appl. Mech. Eng., vol. 19, pp. 59-98, 1979

S. V. Mattsson, "On Modeling of Heat Exchanger in Modelica", Proc. of the $9^{\text {th }}$ European Simulation Symposium, ESS'97, Passau, Germany, Oct 19-23, 1997

Modelica Association, Modelica Language Specification version 3.3 Revision 1, 2014

C. M. Park and A. Gomezplata, "Axial dispersion in a tubular flow vessel with bends," Can. J. Chem. Eng., vol. 49, no. 2, pp. 202-206, 1971

S. V. Patankar, Numerical heat transfer and fluid flow. CRC press, Taylor and Francis group, 1980

Scilab Enterprises. Scilab: Free and Open Source software for numerical computation, 2015. Available from: http://www.scilab.org

R.K. Shah and D.P. Sekulić, "Fundamentals of heat exchanger design" John Wiley \& Sons, Inc., Hoboken, New Jersey, 2003.

D. Tenchine and P. Gauthé, "Occurrence of thermal stratification in sodium cooled fast reactor piping," Nucl. Eng. Des., vol. 274, pp. 1-9, Jul. 2014

S. Velut, P.-O. Larsson, J. Windahl, L. Saarinen, and K. Boman. "Short-Term Production Planning for District Heating Networks with JModelica.org." Proc. of the 10-th International Modelica Conference, Lund, Sweden, March 2014. DOI: 10.3384/ECP14096959

M. Wetter, Wangda Zuo, Thierry S. Nouidui and Xiufeng Pang, Modelica Buildings library, Journal of Building Performance Simulation, 2014 Vol. 7, No. 4, 253-270, DOI: 10.1080/19401493.2013.765506.

E. B. Wylie and V.L. Streeter, "Fluid Transients", McGrawHill Inc, New York, New York 1978 